\title{
The Impact of Rubbernecking on Urban Freeway Traffic
}

\author{
Jonathan Masinick ${ }^{1}$, Hualiang Teng ${ }^{2}$, Nesley Orochena ${ }^{2}$ \\ ${ }^{1}$ Traffic Engineer at Michael Baker Corporation, Richmond, USA \\ ${ }^{2}$ Department of Civil and Environmental Engineering and Construction, University of Nevada, Las Vegas, USA \\ Email: jon@masinick.com, Hualiang.Teng@unlv.edu, Nesley.Orochena@gmail.com
}

Received August 8, 2013; revised September 16; 2013; accepted October 12, 2013

Copyright (C 2014 Jonathan Masinick et al. This is an open access article distributed under the Creative Commons Attribution License, which permits unrestricted use, distribution, and reproduction in any medium, provided the original work is properly cited. In accordance of the Creative Commons Attribution License all Copyrights @ 2014 are reserved for SCIRP and the owner of the intellectual property Jonathan Masinick et al. All Copyright (C) 2014 are guarded by law and by SCIRP as a guardian.

\begin{abstract}
An incident influences traffic not only in the incident direction but also in the opposite direction. There has been a research on the influence of incidents on the traffic in the incident direction. However, the research relating to the influence on the opposite direction of traffic is rare. In this study, the impact of incidents on the traffic in the opposite direction was investigated, focusing on identifying the factors that influence the likelihood and traffic delay of rubbernecking. In the investigation, a database consisting of incident information, traffic and other related variables was developed. For each incident in the database, it determined whether the impact of rubbernecking on the opposite direction traffic was significant. Factors that influenced the likelihood of rubbernecking were identified based on developing a binary logit model. Traffic delays in the opposite direction due to accidents were also calculated. A linear regression model was developed from which the influencing factors on traffic delay were identified. Based on the study on the likelihood of rubbernecking and the associated traffic delay, effective countermeasures were developed.
\end{abstract}

\section{KEYWORDS}

Rubbernecking; ITS Data; Regression Modeling

\section{Introduction}

Freeway incidents cause major congestion throughout the United States every year. These incidents are often vehicle-vehicle accidents, which often cause major backups along freeways, sometimes for miles. These overcrowded gridlock situations cost travelers significant time and money. Other costs incurred due to freeway incidents include increased potential for secondary accidents, additional wear and tear on vehicles, and environmental pollution. Historical statistics show that more than $50 \%$ of urban freeway congestion is related to incidents. Reducing the amount of congestion with various Intelligent Transportation Systems (ITS) or by other methods is an important area of research by which time, money, and even lives can be saved. In the past, research has focused on determining and modeling the impacts of incidents in the direction of traffic where incidents occur. The results from this research can be used to determine such system performance measures as delays, capacity reduction, and travel times.
Although the modeling of incident traffic in the same direction is important, it deals with only half of the traffic problem. Incidents also have an impact on the opposite direction of traffic. Even though there are no lane blockages in the opposite direction of an incident, there are reasons to believe that an impact on traffic exists. This impact is due to rubbernecking. According to the Merriam-Webster online dictionary, "rubbernecking” means "to look about or stare with exaggerated curiosity" [1]. Individuals driving in the opposite direction of an accident often are distracted by the incident. It is the curiosity about the event that leads to distraction, and then causes a reduction in vehicle speeds. This reduction in vehicle speeds begins to create congestion. Although a significant part of rubbernecking is attributed to various human factors, there are other factors, such as the presence of barriers that influence the type of rubbernecking involved.

The goal of this study is to investigate the impact of traffic in the opposite direction of travel from a vehicle 
incident, based on archived Intelligent Transportation Systems (ITS) data. Due to limited resources, this study focused on one type of incident: accidents. To accomplish this investigation, the following objectives were achieved:

1) Determine the likelihood of rubbernecking in the opposite direction of accidents, and

2) Identify the factors that influence the impacts of the rubbernecking on traffic delay.

To determine the likelihood of rubbernecking, accidents that caused rubbernecking in the opposite direction were identified from the total set of incidents. Based on the pool of the accidents for which the impacts due to rubbernecking were identified, the likelihood of rubbernecking was modeled by developing a binary logit model. Given certain factors, such as the time period of an accident and the existence of median barriers, the likelihood of rubbernecking can be calculated by using the binary logit model. In addition, traffic delays caused by rubbernecking also were calculated. Linear regression models were developed for the calculated delays. Based on the linear regression models, the influencing factors were identified correspondingly. By summarizing the influencing factors on the likelihood of rubbernecking and the associated traffic delay, mitigation measures were recommended.

The area of interest during this study was the freeway system in the Hampton Roads area of Virginia (see Figure 1). This freeway system consists of approximately 10 miles of Interstate 64 from I-564 south to Indian River Road, and also Interstate 264 eastbound from the I-64 interchange. Incidents and their associated data have been collected by the Hampton Roads Smart Traffic Center (HRSTC) and archived by the University of Virginia's Smart Travel Lab. This study limited the incident type and time frame examined to vehicle accidents in the year 2000.

The remaining parts of this paper are organized as follows. The first section presents previous research done on the various issues related to this study. The second section describes the methodology used in this study. The third section shows the analysis of the results, including regression models and the interpretation of the results. The last section is devoted to conclusions and recommendations.

\section{Background Review}

An incident is a traffic event that has an impact on traffic conditions. Incidents come in many forms, including disabled vehicles, abandoned vehicles, various spills and debris, and environmental events (weather). Probably the most influential involves vehicle accidents. All these types of incidents decrease traffic flow and add additional congestion to the already crowded urban freeways. This in turn causes the level of service to decrease and

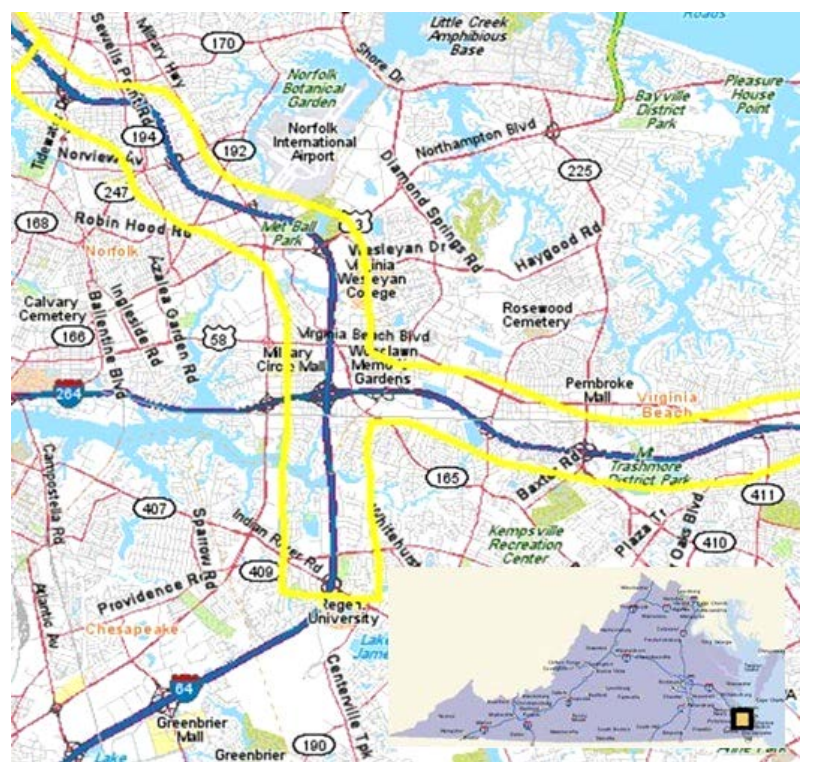

Figure 1. Area map of the Hampton roads freeway system.

results in additional incidents. Previous research has been done on traffic impacts of incidents, incident management, incident prediction, and other topics pertaining to these random events. Although the information gathered for these studies is typically for traffic in the same direction as incidents, review of this information is still valuable for this current study.

\subsection{Rubbernecking Effects}

Rubbernecking is a result of a human response to the surroundings, such as freeway signs, scenery, billboard ads, and many other visual "eye-candy". From a traffic operations standpoint, rubbernecking is a serious issue that can sometimes create traffic congestion and even traffic incidents. In addition, the attention of the driver is focused on these surroundings; therefore, less attention is focused on the roadway. This makes rubbernecking a safety issue as well as a traffic congestion issue.

A study by the Crash Investigation Team of the Transportation Safety Training Center at Virginia Commonwealth University revealed that rubbernecking was the leading cause of vehicle crashes [2]. These rubbernecking accidents were not caused by landmarks or other scenery; they were caused by drivers looking at other vehicle crashes and other roadside traffic incidents. Rubbernecking caused by vehicle crashes and other incidents accounted for $16 \%$ of all vehicle crashes, while the total number of "outside the car" distractions accounted for $35 \%$. There has been research performed that calculated the effects of rubbernecking on traffic in the same direction of travel as incidents. These effects are due to rubbernecking events occurring in adjacent lanes and shoulders. 
Drivers cannot be distracted by events or objects they cannot see. To mitigate rubbernecking in the opposite direction, barriers that block vision to opposite direction traffic conditions are used. The Hampton Roads freeway system, consisting of I-64 and I-264, implements a variety of barrier techniques (see Figure 2). Certain segments along the freeway system only have guardrails and a grassy median dividing the freeway traffic. Certain sections of the Hampton Roads freeway have standard $107 \mathrm{~mm}$ (42-inch) concrete barriers, while other sections have double-stacked concrete barriers. By using data regarding the different types of barriers on roadway segments, it is possible to investigate their relationships with the impact that rubbernecking has on traffic conditions in the opposite direction. The derived information could help develop mitigations to reduce rubbernecking impacts on opposite direction traffic conditions.

\subsection{Traffic Delays}

"Congestion delay" is referred to as the difference between actual travel time and the free-flow time on a section of freeway [3]. It can be determined for a wide variety of traffic situations, such as freeway and arterial systems. In freeway systems, delay is often thought about in terms of "recurrent" and "non-recurrent" delays [4].

Incident-induced delays have been calculated using a variety of methods. Morales developed a cumulative volume approach to calculating freeway delays [5]. In this approach, two cumulative volume curves, one for arrival and the other for departure at an incident site, are plotted on a time axis. The area between these two curves represents the delay due to an incident.

Suggestions have been made to adjust one or both of these curves. Daganzo proposed a "virtual" arrival curve be used to determine delays [6]. This virtual arrival curve is a translation of the actual arrival curve, based on the "number of items that would have been seen directly upstream of the restriction" by the beginning of the incident duration [6]. The actual arrival curve is translated to the right by a value of $\tau$, representing the travel time between observers, or stations. This new method of determining delays is just one of the recent methods used.

Al-Deek et al. developed a new method that improved upon Morales' approach by looking at delays in time slices [7]. They incorporated vehicle speeds in conjunction with traffic volumes to develop a delay formula. Assumptions they made include the following:

- Traffic speed and volume data are determined from the loop stations on a roadway segment; these data are homogeneous throughout the segment.

- Incident delay is calculated with respect to a reference (or base) average speed that reflects normal conditions, which may or may not be congested. The reference speed represents a historical speed profile,

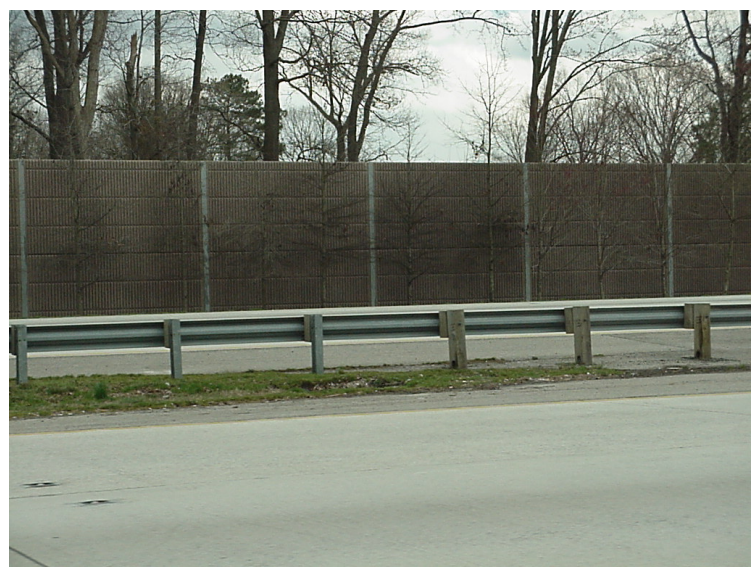

(a)

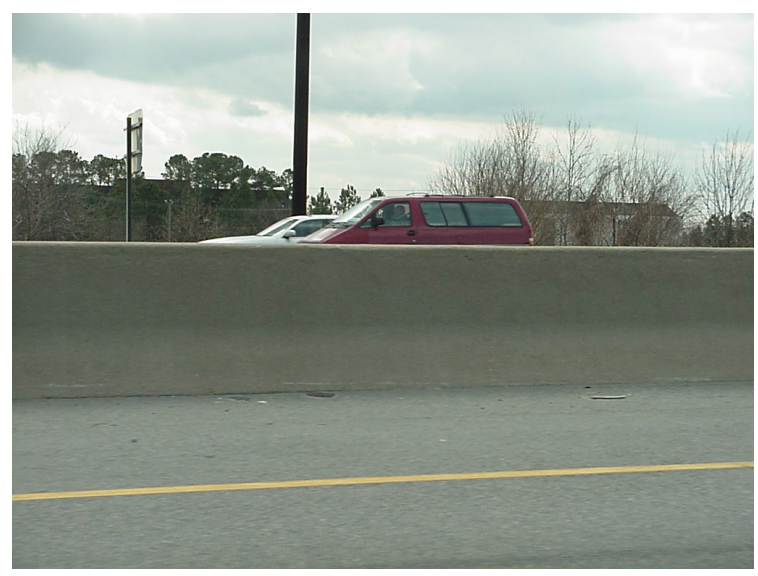

(b)

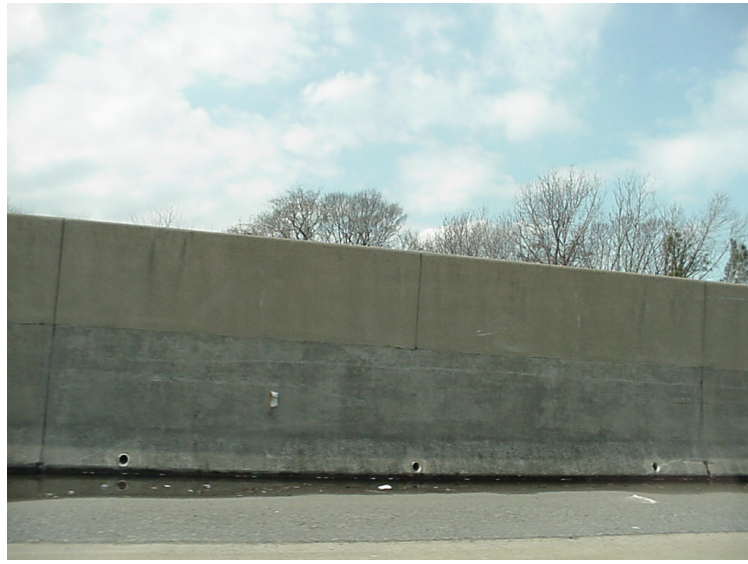

(c)

Figure 2. (a) Barrier guardrail system on a section of roadway on I-64. (b) A standard concrete barrier on I-264. (c) A double-stacked concrete barrier on I-64.

which may be used to segregate, or distinguish between both incident and non-incident (recurring) congestion.

A drawback to this approach is that it required oneminute speed averages. Smaller interval averages of less than one minute could lead to "noisy" data, while larger 
intervals of greater than one minute do not allow for accurate estimation of queue boundaries. Different from the queuing diagram approach, where incident duration, capacities before and after an incident, and traffic demand are used to calculate delay, the incident delay is determined using the time-slice method. The individual slices are summed up to form the total delay.

The approach proposed by Qi and Teng is an extension of Morales' approach [8]. By integrating equations for frequency, duration, and lane blockage, each with their influencing factors, into a delay formula, the impact of these factors on delay can be estimated.

In addition to approaches based on queuing diagrams and real-time traffic data, computer simulation is another effective way in modeling traffic delays during incidents.

\section{Methodology}

\subsection{Traffic and Incident Data}

Data for the Hampton Roads freeway system operated by the HRSTC were collected for this study. There is an incident management system in the HRSTC by which incidents are documented into a database; this includes a variety of information regarding the incident. Loop detectors are installed along the freeway system in the HRSTC. From these detectors, real-time traffic data are collected and sent to the Center. Data of both the incident and the loop detector are accessible through the Smart Travel Lab at the University of Virginia, in Charlottesville.

Specifically, the incident database was retrieved from the Smart Travel Lab's hr.incident table. This table contains information on each incident, and includes subtables with additional information. The information on incidents include incident identification number, incident begin time (including date/time in MM/DD/YYYY HH24: MI format), incident duration (in minutes), incident type, weather, detection source, and a brief description of the incident. Sub-tables include such information as the roadway of occurrence; direction; location; number of lanes and shoulders blocked; and information about the vehicle(s) involved, such as make, model, and color.

Although useful in other studies, some of this information is not pertinent to this study. The relevant information used in this study included the incident identification number, incident begin time, roadway, direction, location, duration of incident, weather, number of lanes, shoulders blocked, and description. Incidents that took place in the year 2000 were pulled from the hr.incident table. During this time period, available incident types included abandoned vehicles, vehicle accidents, bridge incidents, debris, disabled vehicles, and "other". It was decided that impacts due to rubbernecking would most likely only occur during vehicle accidents. The acquired incidents were then filtered to include only incidents whose type was designated "accident".

Traffic data were collected based on the date, time, and location of each incident that was included in the database. Note that the exact sites of the incidents cannot be readily known from the location code of the incident in the hr.incident database. Each location code identifies a section of roadway typically two miles long and having three or four detector stations 1.5 miles apart from each other. Thus, traffic data had to be collected for all stations within the location code of the incident. Total volumes, average speeds, and average occupancy were collected for an extended period, starting from one hour before the incident beginning time and ending at one hour after the duration of the incident. This period accounts for the time period when traffic is operating normally before the incident and also when traffic is recovering and once again operating normally after the duration of the incident. Collecting data for this extended period of time ensured that the full effects of the incident were captured.

\subsection{Determination of Incident Location and Significance of Rubbernecking Impacts}

The traffic and incident information for one incident was compiled into one single spreadsheet. In order to determine whether the incident had significant impacts on traffic in the opposite direction, visual examination was conducted of an Occupancy vs. Time of Day, an example of which is shown in Figure 3. In this study, such plots were created for both the incident direction and the opposite direction. The plots showed whether a significant increase or decrease in occupancy was present in both travel directions. As mentioned before, the HRSTC incident database only gave a vague indication as to the location that the incidents took place. The "location code" indicates the section of roadway, consisting of multiple stations. There is no documentation as to between which stations the incident took place.

Using Figure 4, the approximate location of each accident can be determined based on observing the patterns of the changes in occupancies. Specifically, it was perceived that the immediate upstream station from each accident would have the earliest and largest occupancy impact. Subsequent upstream stations should also show an impact, but at a later time, due to the backward-moving shockwave. The occupancy plot of multiple stations, shown in Figure 4, indicates the immediate upstream station of the incident. It can be seen that the impacts of increased occupancy reaches Station A prior to reaching Station B and Station C. This pattern shows that Station $\mathrm{A}$ is the immediate upstream station of the accident.

Precautions were taken in dealing with these traffic 


\section{Incident Occupancy}

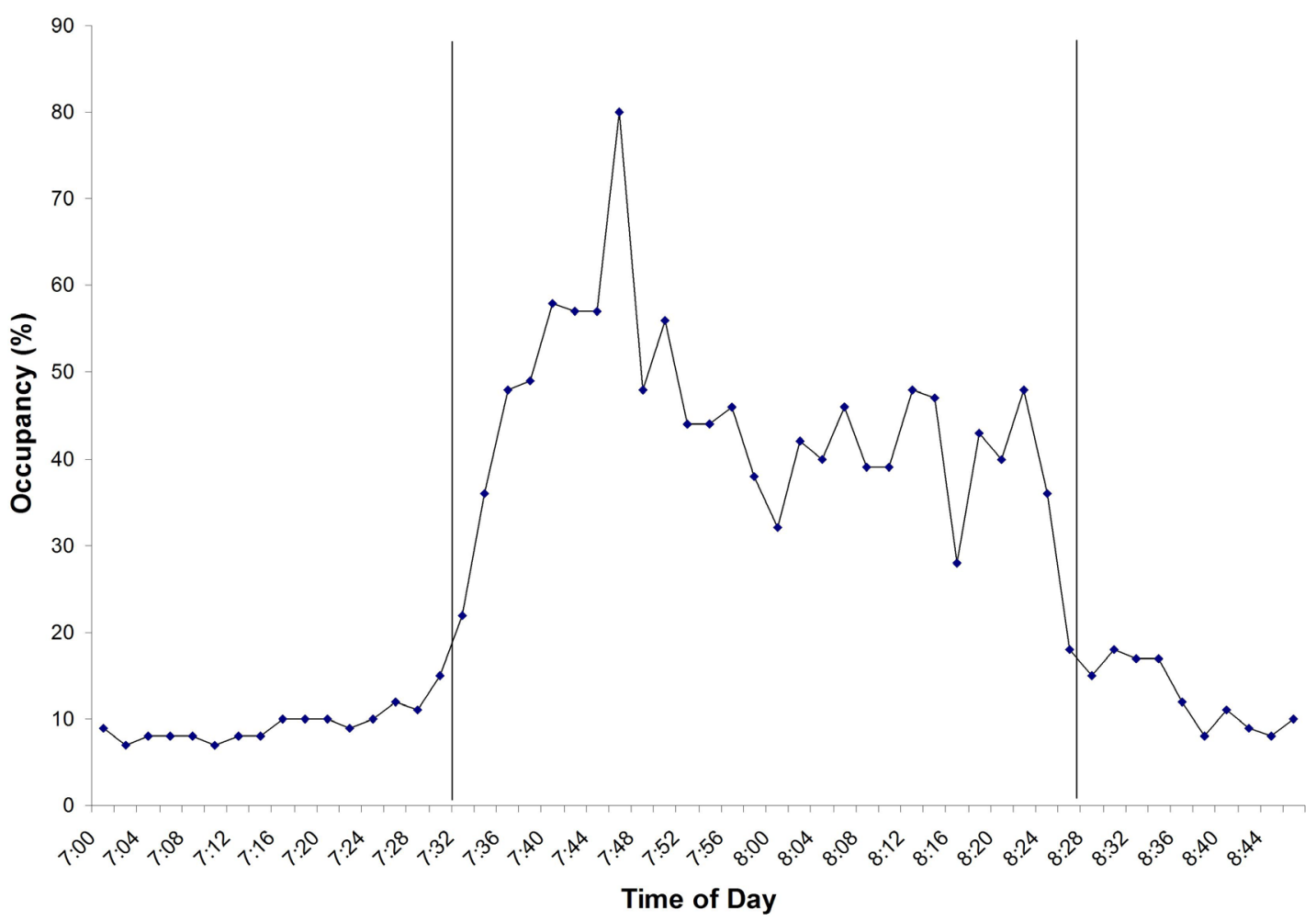

Figure 3. Examples of incident occupancy.

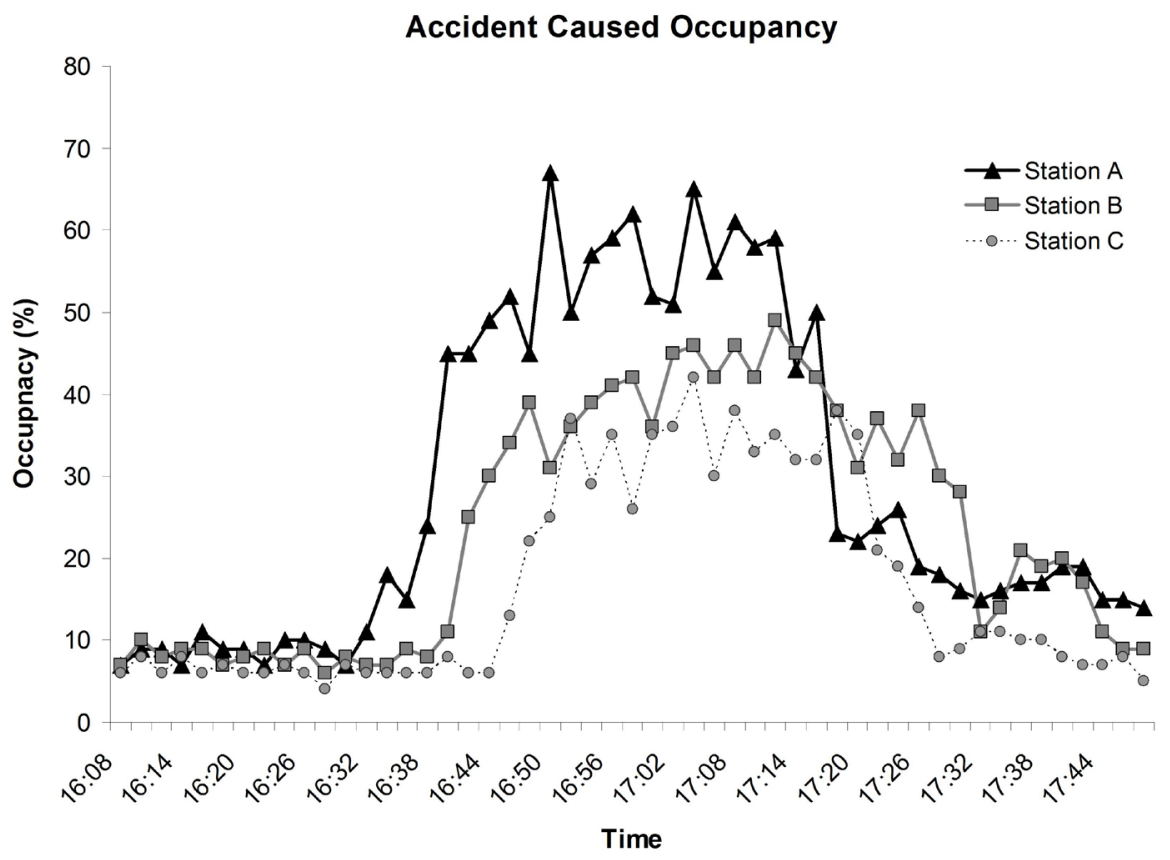

Figure 4. Incident-caused occupancy at multiple stations.

data. Multiple incidents occurring in the same time periods can give misleading results. Once the accident database was developed and traffic data were collected, it was checked whether the accidents were isolated events having no other incidents affecting the target area. Queries were run to determine whether additional incidents were involved during the time period and location of the incidents. Multiple incidents occurring during these ac- 
cident times were not used in the analysis.

\subsection{Using the Binary Logit Model to Evaluate}

Whether an accident causes impacts due to rubbernecking is a binary variable. To identify the factors that are associated with such a binary variable, a binary logit model can be developed. According to the binary logit model, the "utility" for an accident to cause rubbernecking impact on the opposite direction traffic can be written as:

$$
U_{\text {in }}=\beta^{\prime} \mathbf{x}_{\text {in }}+\varepsilon_{\text {in }}
$$

where $\beta$ represents a vector of coefficient for explanatory variables included in the vector $\boldsymbol{x}_{i n}$, and $\varepsilon_{i n}$ is denotred as the error of the "utility", $U_{i n}$. The maximum likelihood estimation method was used to determine the coefficients of the model. The likelihood of rubbernecking during an accident can be calculated as follows:

$$
P(i=1)=\frac{\mathrm{e}^{U_{i n}}}{\mathrm{e}^{U_{i n}}+\mathrm{e}^{U_{0 n}}}
$$

where " 1 " represents rubbernecking and “ 0 ” represents no impact.

\subsection{Congestion Delay Calculations and the Identification of Influencing Factors}

In this study, incident delay was derived based on the cumulative volume plots, and upstream and downstream station volumes were used. As shown in Figure 5, the area between the two curves represents the increased delay due to an accident. In this study, this area is measured by taking the integral of the difference of the curves over the duration of the impact of the accident. This duration should not be confused with the database documented as "duration". The "duration" database involves the time between the arrivals of service vehicles and the complete clean-up of the incident. The duration used in the integral should be viewed as the duration of the impact of the incident and the recovery from the incident. The integral can be written as follows:

$$
\int_{T_{1}}^{T_{2}}[A(t)-D(t)] \mathrm{d} t
$$

where $A(t)$ and $D(t)$ represents the cumulative arrival and departure curves, respectively. Note that using the integral to calculate delays requires the functions of the cumulative curves, which are unavailable in this case of traffic delay calculation. This problem was solved by using the properties of the area function, a routine used to estimate areas under complex functions. In order to find the area under a curve, smaller rectangles can be made. These smaller rectangles are added together to determine the area under the curve. The time-step used in this study

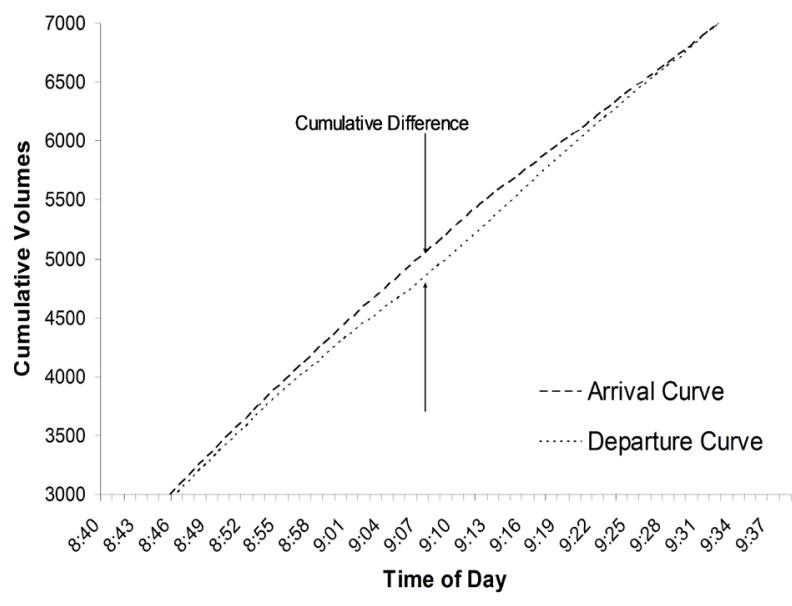

Figure 5. Example of cumulative arrival and departure curves.

corresponded to the quality of data collected. Since the study used one-minute aggregate data, the respective time-step for the delay integral was one minute. The yaxis direction represents the difference in the arrival and departure curves of the incident. The $\mathrm{x}$-axis represents the time-step or data collection interval.

The additional development of a "virtual arrival curve" (5) was not appropriate in this study. The travel time $\tau$ between $400 \mathrm{~m}$ (1/4-mile) or $530 \mathrm{~m}$ (1/3-mile) station gaps, based on $95 \mathrm{~km} / \mathrm{hr}$ (60 $\mathrm{mph}$ ) free flow speeds, would be approximately 15 - 20 seconds. A 20-second lateral translation of the arrival curve would not be a significant change to the curves.

In this study, a linear regression model was used to identify the factors that significantly determine the amount of traffic delay to the opposite direction traffic. In the modeling, the measure of traffic delay served as response variables, and the characteristics that may lead to delay were the independent, or predictor, variables. These variables included the duration of the incident, weather, number of lanes and shoulders blocked, peak and non-peak hours, day/night, weekend/weekday, and visual barriers. In general, the linear regression model takes the form of:

$$
Y=\beta_{0}+\beta_{1} X_{1}+\beta_{2} X_{2}+L+\beta_{k} X_{k}
$$

where $y$ denotes delay, $X$ represents influencing variables, and $\beta$ is the coefficient.

\section{Results and Analysis}

\subsection{Incident Data}

Initially, incident data were collected for the years 2000 and 2001. These incidents were filtered only to include “accident"-type incidents. It was soon brought the attention of the researchers that traffic data from the Hampton Roads Smart Traffic Center were missing for a good part 
of year 2001. Thus, 2001 accident and traffic data was removed from the database, leaving only year 2000 accident and traffic data.

In summary, 36,769 total incidents occurred in the Hampton Roads freeway system in the year 2000, with 2175 being accidents. During that year, accidents accounted for $5.9 \%$ of the total incidents. Many of these accidents did not provide any location data, so they were excluded. The analysis conducted in this study was based on the 840 accidents with sufficient incident data information.

\subsection{Likelihood of Rubbernecking}

By visually observing occupancy behaviors in all 840 documented accidents, significant impacts could be distinguished. Significant impacts due to accidents are fairly easy to make out. Typically, a sharp increase of occupancy occurs soon after the accident begins. This increased occupancy usually is held fairly constant for the duration of the incident. After the incident has cleared, the occupancy returns to its normal values. Out of the 840 accidents in the year 2000, the results of significant impacts can be seen in Table 1 .

This table shows that 201 out of the 840 accidents had an impact on traffic occupancy in the same direction as the accident. 102 out of the 840 showed significant changes in occupancy for the opposing traffic. Out of these accidents, 84 showed a significant impact in both directions. As seen in the table, this situation makes up $10 \%$ of the total number of incidents.

Table 2 lists the results of the binary logit model. It can be seen that four variables significantly influenced whether an accident impacted the traffic in the opposite direction: peak/non-peak hours, weather, presence of barriers, and weekday/weekend.

The coefficient for variable "peak" was negative, which implies that an incident that occurred in peak periods was less likely to cause a rubbernecking impact to the opposite direction of traffic. This might be reasonable because motorists are in a rush of homeward-bound or work-bound travel. Under this condition, the curiosity of the motorists to know the accidents in the other direction may be under a certain amount of control.

The coefficient for "weather" (rain/snow/ice) was negative. This indicates that an accident which occurred in rain might be less likely to attract attention from motorists traveling in the other direction. It might be reasonable to expect that the bad weather demanded more attention of the motorists on their travels, with less chance for them to care about the events happening in the other direction.

The coefficient for "weekday" was positive. This suggests that an accident which occurred during a weekday would be more likely to cause rubbernecking in the op-
Table 1. Statistics of significant impacts of occupancy due to accidents.

\begin{tabular}{ccc}
\hline Category & $\begin{array}{c}\text { Number of } \\
\text { Significant Impacts }\end{array}$ & $\begin{array}{c}\text { Percentage } \\
(\%)\end{array}$ \\
\hline Total Accidents & 840 & 100 \\
Same Direction as Acident & 201 & 23.9 \\
Opposite Direction as Accident & 102 & 12.1 \\
$\begin{array}{c}\text { Significant Impact in both SAME } \\
\text { and OPPOSITE Directions }\end{array}$ & 84 & 10.0 \\
\hline
\end{tabular}

Table 2. Results of a binary logit model.

\begin{tabular}{cccc}
\hline Variable & Coefficient & Standard Error & t-test \\
\hline Constant & -4.39220546 & 0.65932825 & -6.662 \\
Peak & -3.27492185 & 0.49187300 & -6.658 \\
Weather & -0.97968397 & 0.42100950 & -2.327 \\
Barrier & -0.47736199 & 0.43069356 & -1.108 \\
Weekday & 5.17244375 & 0.60798673 & 8.5070 \\
Log Likelihood Function & & -130.1868 & \\
Restricted Log Likelihood & & -281.3884 & \\
Chi Squared & & 302.4033 & \\
Degrees of Freedom & & 4 & \\
Number of Observations & & 840 & \\
\hline
\end{tabular}

posite direction than if it occurred in weekends. This may be due to the high volume of traffic during weekdays versus weekends. Under high-volume conditions, the potential number of motorists to rubberneck would be more than that under the low-volume conditions.

As far as the factor of "barriers" was concerned, the coefficient was negative. This implies that the presence of barriers at an accident scene decreased the likelihood of rubbernecking in the opposite direction. This may be because the barriers blocked the motorists' view of accidents in the other direction. In the Hampton Roads area, there were two types of barriers, each with different heights. Each of them can only block the views of a certain portion of motorists. Thus, the significance of the variable "barrier" cannot be demonstrated as strong as expected. Considering this possible error, the result was accepted as reasonable.

The frequencies of the actual and predicted outcomes of the binary logit model are presented in Table 3 . Based on 840 accidents, the binary logit model predicted which accidents would impact opposing traffic. Out of the 84 accidents that showed significant impacts on opposing traffic, 70 (or $83.3 \%$ ) were selected by the binary logit model to have such an impact. The remaining 14 accidents were deemed insignificant. The success of the model is also demonstrated by the total number of significant and insignificant impacts that were predicted. The impacts of 783 (93.214\%) out of the total number of 
Table 3. Prediction results for a discrete choice model.

\begin{tabular}{|c|c|c|c|}
\hline \multirow[t]{2}{*}{ Actual } & \multicolumn{2}{|c|}{ Predicted } & \multirow[t]{2}{*}{ Total } \\
\hline & 0 & 1 & \\
\hline 0 & 713 & 43 & 756 \\
\hline 1 & 14 & 70 & 84 \\
\hline Total & 727 & 113 & 840 \\
\hline
\end{tabular}

accidents had been successfully predicted using this model.

\subsection{Traffic Delay Calculations and Modeling}

Traffic delays in the opposite direction were calculated for the 84 accidents that showed significant impact on traffic in both the same and opposite directions. These calculations were possible using cumulative volume plots, as described in the methodology section. Delay calculations resulted in a range of $3.6 \mathrm{veh} \cdot \mathrm{hr}$ to $590.0 \mathrm{veh} \cdot \mathrm{hr}$. A histogram of the delay is presented in Figure 6. The histogram shows that lower delays are prevalent. Specifically, $78.5 \%$ of the delays fall between 0 and $150 \mathrm{veh} \cdot \mathrm{hr}$. This indicates that major congestion delay was not common. Only $12 \%$ of the delays were over $200 \mathrm{veh} \cdot \mathrm{hr}$.

In modeling the delay, using linear regression model, it was realized that delay cannot be used directly as the dependent variable. This is because the calibrated linear regression model cannot guarantee that a delay with a positive value will be produced; this would not be convenient if the model is used for forecasting. A regular approach to dealing with this situation is to use a natural log transformation of delay as the dependent variable in regression. After the transformation, it was found that the natural log transformation of the delays was better distributed for normal distribution than for that without the transformation. Therefore, the regression analysis was performed on this newly adopted transformation. In the calibration of the linear regression model, independent variables used in this analysis include:

- Duration: the time period required to clear an incident,

- Weekday/Weekend: whether an accident happened during a weekday or weekend,

- Peak: whether an accident happened during an AM or PM peak rush,

- Weather: inclement or clear weather,

- Barrier: presence of barriers,

- Day/Night: whether the accident happened during the day or at night,

- Lanes Blocked: number of lanes blocked by accident,

- Shoulders Blocked: number of shoulders blocked by accident, and

- Volume/Capacity Ratio: volumes before the accident versus the capacity on the roadway segment where an incident occurred.
A model was calibrated, and results are presented in Table 4. It can be seen from the table that the coefficient of the variable incident duration was positive. This implies that accidents with longer duration will have greater delays associated with them. This result is consistent with our expectation. The variable "barrier" is a binary variable where " 0 " means no barrier was present at an accident site and " 1 " indicates the presence of a barrier.

As shown in Table 4, the coefficient "barrier" was negative. This means that less delay would be incurred to the traffic in the opposite direction if barriers were present at an accident site. This result is consistent with the result for the likelihood of rubbernecking.

The coefficient of V/C ratio, volumes before the incident versus the capacity of the roadway segment where an incident occurred, was positive. This indicates that higher V/C values account for greater delays; this also is consistent with our expectation.

\section{Conclusions and Future Study Needs}

\subsection{Conclusions}

This study was a first attempt to evaluate the impact of rubbernecking of accidents on traffic in the opposite direction, based on archived data of traffic and accidents. Two models were developed for determining the likelihood of the occurrence of rubbernecking as well as traffic delays caused by rubbernecking. The data indicated that about $10 \%$ of accidents were caused by rubbernecking, and that the average delay caused by rubbernecking in the opposite direction was $107 \mathrm{veh} \cdot \mathrm{hr}$. These statistics indicate that the impact of rubbernecking is significant, and that certain mitigation measures have to be taken.

Based on the interpretation of the results of the regression models, it can be concluded that the likelihood of rubbernecking is influenced by peak periods, weather, presence of barriers, and weekday travel; the delay is influenced by duration, presence of barriers, and the volume and capacity ratio. Based on these identified factors, countermeasures can be developed that target the time period and locations specified by these variables.

Barriers are an effective way to reduce the likelihood of rubbernecking in the opposite direction and the delay caused by the rubbernecking. This conclusion was drawn upon the coefficients of the variable "barrier" in the models for the likelihood of the occurrence of rubbernecking and traffic delay. The statistical significance of these coefficients in these models implies that barrier is a significant contributor to the occurrence of rubbernecking as well as the traffic delay in the opposite direction caused by the rubbernecking. Intuitively, installation of barriers can be thought of as a direct way to mitigate the occurrence of rubbernecking. Because it is costly if concrete barriers are installed along all the highway systems, 


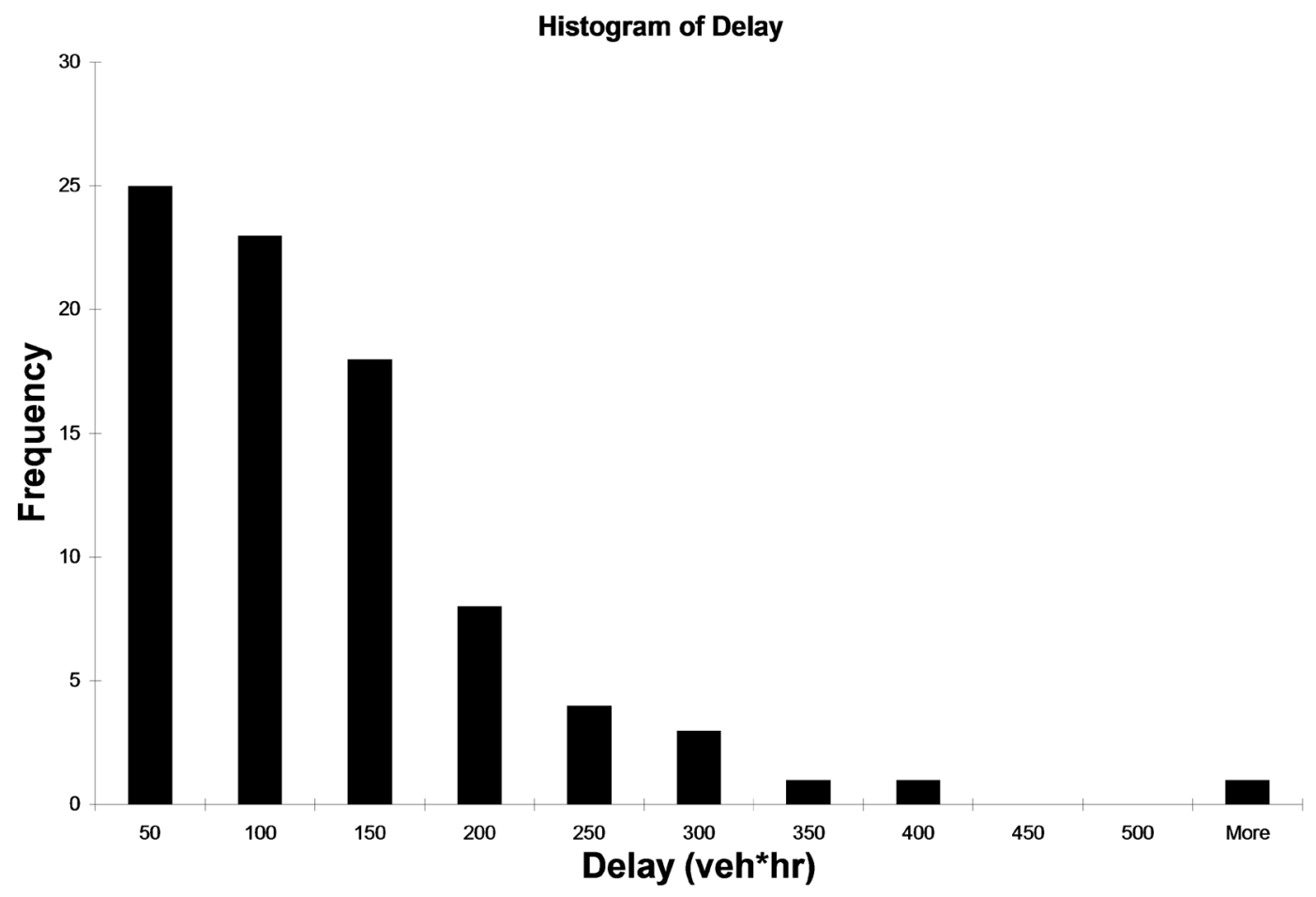

Figure 6. Histogram of the frequency of delay.

Table 4. Results of a binary logit model.

\begin{tabular}{cccc}
\hline Variable & Coefficient & Standard Error & t-test \\
\hline Constant & 7.24118149 & 0.46588171 & 15.543 \\
Duration & 0.01963081 & 0.00218229 & 8.995 \\
Barriers & -0.25688358 & 0.18161164 & -1.414 \\
VolCap & 0.72782444 & 0.56328168 & 1.2920 \\
Degrees of Freedom & 80 & & \\
R-Squared & & 0.5301680 & \\
Adjusted R-Squared & & 0.5125493 & \\
Number of Observations & & 84 & \\
\hline
\end{tabular}

such cost-effective barriers as portable screens can be investigated.

\subsection{Future Research}

Rubbernecking is one of the major problems in highway systems. The methodology and results of this study can act as a basis of future research on rubbernecking, and also could be used to spark interest in issues related to rubbernecking and their impacts. The following issues have been identified for future research: the quality of incident and traffic data, statistical modeling, and human factor characteristics.

Regarding the quality of incident and traffic data, more accurate information about incidents should be collected. In order to successfully utilize the incident data, locations of incidents must be exact. A more accurate identifica- tion of the incident's location would aid in determining upstream and downstream stations required for many traffic measures. Currently, the descriptions of incidents in the database often are short and do not contain useful information. A detailed description would improve the usefulness of this data.

Also, more information about the accidents, such as the types and number of vehicles involved, should be collected. By having this information available, it is possible to identify whether this information contributes to rubbernecking in the opposite direction. As a result, the factors at in accident scenes that attract motorists to slowdown, causing rubbernecking, could be more exactly identified.

An effort should be made to model the impact of barrier height on the likelihood of rubbernecking and traffic delay. The results in the likelihood model indicated that "barrier" was not as significant as other variables. Also, the variable "barrier" was set as binary; therefore, the height of barrier could not be investigated. To derive information that is more helpful in installing barriers that prevent rubbernecking, it would be beneficial to have a clear understanding of the relationship between the barrier's height and the impact of rubbernecking.

Finally, the role of human factors on rubbernecking needs to be investigated. As indicated in the analysis of this study, motorists during peak periods tend to create less rubbernecking than during other periods. This suggests that human factors play a role in the causes of rub- 
bernecking and its effects. By understanding the significance of human factors, the issue of rubbernecking may be better addressed.

\section{REFERENCES}

[1] 2013.

http://www.meriam-webster.com/dictionary/rubberneck,r etrieved

[2] Crash Investigation Team, "Driver Inattention and Driver Distraction Study,” Special Report \#15, Transportation Safety Training Center, Virginia Commonwealths University, 2001.

[3] R. W. Hall, "Non-Recurrent Congestion: How Big Is the Problem? Are Traveler Information Systems the Solution?” PATH Program, Institute of Transportation Studies, University of California, 1992.
[4] J. Lindley, "Urban Freeway Congestion: Quantification of the Problem and Effectiveness of Potential Solutions," ITE Journal, Vol. 57, No. 1, 1987, pp. 27-32.

[5] J. M. Morales, "Analytical Procedures for Estimating Freeway Traffic Congestion,” Public Roads, Vol. 50, No. 2, 1986, pp. 55-61.

[6] C. Daganzo, "Fundamentals of Transportation \& Traffic Operations,” Elsevier Science Ltd., Oxford, 1997.

[7] H. M. Al-Deek, A. Garib and A. E. Radwan, "Methods for Estimating Freeway Incident Congestion, Part II: New Methods," Paper Submitted to the ASCE Journal of Transportation Engineering, 1994.

[8] Y. Qi and H. Teng, "Integrated Modeling Approach to Total Incident Delay,” Transportation Research Record, 1895, pp. 46-54. http://dx.doi.org/10.3141/1895-07 